Ann. Zootech., I979, 28 (2), 23I-234.

\title{
Adoption d'un deuxième veau par des Vaches plusieurs jours après la mise-bas
}

\author{
P. LE NEINDRE et J.-P. GAREL \\ Laboratoire de la Production de Viande \\ Centre de Recherches de Clermont-Iierrand, I.N.R.A., \\ Theix, St-Genès-Champanclle, 631 Io Beaunont (France) \\ Domaine Expérimental de Mavcenat, S.E.I., \\ Domaine de la Borie, I.N.R.A., I5330 Marcenat (France)
}

\section{Résumé}

Afin de favoriser l'adoption d'un second veau par des vaches de race Salers quatre jours après le vêlage, nous avons essayé de perturber les vaches en les masquant et en badigeonnant les veaux avec de l'huile de pied de bœuf. Trois lots sont comparés:

I) le second veau est présenté sans précaution lors d'une tétée;

2) la vache est masquée du vêlage à 8 jours. Le veau de la mère et le second veau sont enduits d'huile de pied de bouf : le veau de la mère de la naissance au huitième jour, le second veau du quatrième au huitième jour;

3) la vache est masquée et les 2 veaux sont enduits d'huile de pied de bœuf du quatrième au huitième jour.

Les adoptions ont été plus fréquentes dans le troisième lot que dans les deux autres. Il semble que les perturbations appliquées lors de la présentation du second veau favorisent son adoption.

Dans une étude antérieure (I,E NEINDRE, Petit et GAREI, I978), nous avons montré, comme KILEY (I976) et HuDSON (I977), que les veaux supplémentaires étaient adoptés en proportion beaucoup plus élevée lorsqu'ils étaient présentés aux vaches immédiatement après la mise-bas que s'ils étaient présentés plus tard. La première méthode est cependant astreignante car l'éleveur doit être présent à la mise-bas et disposer en permanence de veaux supplémentaires. Nous avons donc cherché une technique permettant l'adoption du deuxième veau plusieurs jours après la mise-bas. CROWLEY et DARBY (I97I) préconisent dans ce but 1'utilisation de perturbations visuelles (en masquant la vache) et olfactives (en enduisant les veaux d'huile de pied de bœuf). 
37 vaches de race Salers et leur veau Charolais $\times$ Salers (2) ou Salers (35) ont été utilisés dans cet essai. Les veaux supplémentaires étaient des mâles de race Salers achetés à l'âge de $\delta$ à $I_{5}$ jours.

Le mode de conduite de ces animaux était identique à celui décrit dans une étude antérieure ( $L_{E}$ NEINDRE et GAREL, I 977). Les vaches étaient pendant 1'hiver en stabulation entravée et les veaux tétaient detı fois par jour sous surveillance. Les veaux destinés à l'adoption ont tous été présentés lors d'une tétée le quatrième jour après le vêlage qui a eu lieu en movenne le 5 février. Trois lots de vaches ont été constitués.

- Dans le premier lot (I 3 vaches) témoin, les seconds veaux ont été présentés sous surveillance, mais sans intervention particulière.

- Dans le second lot ( 2 vaches), les vaches étaient masquées et leur veau badigeonné sur tout le corps, une fois par jour, d'huile de pied de bouf pendant 8 jours à partir du vêlage; les seconds veaux étaient badigeonnés de la présentation au huitième jour après le vêlage.

- Dans le troisième lot (I2 vaches), les vaches étaient masquées et leurs 2 veaux badigeonnés d'huile de pied de bœuf de la présentation du second veau au huitième jour après vêlage.

'Trois troupeaux ont été constitués, les animaux de chaque lot expérimental étant répartis également dans chacun d'entre eux. I)ux personnes ont observé ensemble en permanence de l'aube au crépuscule chaque troupeau pendant 2 journées entre le I 8 juin et le 12 juillet. Nous avons retenu trois critères pour juger de la réussite de l'adoption: le nombre de tétées en position parallèle inversée, le nombre de léchages et le nombre total de tétées (I,E NEINDRE et GAREL, I977). Les deux premiers critères sont caractéristiques des relations observées normalement entre la vache et son veau et sont associés en général à une bonne croissance du veau. Trois types de relations mère-jeune sont définis :

- le veau est adopté: il tète en position parallèle inversée et il est léché par sa nourrice au moins une fois à chacune des 2 journées;

- le veau est refusé: il ne tète pas sous sa nourrice;

- le veau est toléré: dans tous les autres cas.

Sur 1'ensemble des animaux observés au pâturage, I6 sur 36 des seconds veaux et 32 sur 36 des veaux des mères sont adoptés (tab1. I). La réussite de l'adoption est différente suivant les traitements. Ians le troisième lot, elle est significativement supérieure à celle observée dans les 2 autres lots (tab1. I). Les perturbations olfactives et visuelles pratiquées en niême temps lors de la présentation du second veau permettent donc de modifier la sélectivité des vaches et de leur faire adopter un deuxième veau. Le masque et le badigeon appliqués dès le vêlage n'apportent aucune amélioration par rapport au lot témoin. I)ans ce cas, les débuts des perturbations ne sont pas simultanés à la présentation du deuxième veau et la vache a peut-être pu distinguer les 2 jeunes d'après leur odeur. Ises traitements expérimentaux ont parfois provoqué non seulement l'adoption du veau supplémentaire mais le refus ultérieur du veau de la mère (lot 2 : I vache; lot $3: 2$ vaches) et même pour une vache du lot $\mathrm{I}$, le refus après le traitement de tout veau. Les seconds reaux lorsqu'ils sont adoptés tètent significativement moins souvent seuls et en position parallèle inversée $(0, I 7$ fois par jour pour les seconds veaux au lieu de r,oo pour les veaux des mères) que les veaux des mères. Cette différence est peut-être liée au passé des 2 types de veaux, les seconds étant plus âgés que les autres et ayant subi dans les premiers jours de leur vie un traitement différent. 


\section{TABLEAU I}

Infiuence d'une perturbation visuelle et olfactive (huile de pied de bauf) sur l'adoption d'un second veau présenté quatre jours après le vêlage

Effect of counter-acting the sense of sight and smell on the adoption of a foster calf four days after calving

\begin{tabular}{|c|c|c|c|c|c|}
\hline \multicolumn{2}{|c|}{$\begin{array}{l}\text { Relations entre la vache et le second veau } \\
\text { Fostor calf-cow relationship }\end{array}$} & $\begin{array}{l}\text { Témoin } \\
\text { Untreated }\end{array}$ & $\begin{array}{l}\text { Masquage } \\
\text { au vêlage } \\
\text { Blindfolded } \\
\text { at calving }\end{array}$ & $\begin{array}{l}\text { Masquage } \\
\text { a } 4 \text { jours } \\
\text { Blindfolded } \\
\text { the fth day }\end{array}$ & $\begin{array}{l}\text { Total } \\
\text { Total }\end{array}$ \\
\hline $\begin{array}{l}\text { Second } \\
\text { veau }\end{array}$ & Adopté (Adopted) . . . . . . . & 2 & $\left(\operatorname{dont}^{4} I^{*}\right)$ & $\begin{array}{c}\text { Io } \\
\left(\text { dont } 2^{*}\right)\end{array}$ & $\stackrel{\text { I6 }}{\left(\text { dont } 3^{*}\right)}$ \\
\hline \multirow{2}{*}{$\begin{array}{l}\text { Foster } \\
\text { calf }\end{array}$} & Toléré (Tolevated) . . . . . . & 6 & 2 & $\mathrm{r}$ & 9 \\
\hline & Refusé $($ Refused). . . . . . . & 5 & $6 * *$ & I & I I ** \\
\hline Total & . . . . & I 3 & I 2 & 12 & 37 \\
\hline
\end{tabular}

$\mathrm{x}^{2}:$ I $5, \mathrm{O} P<$ o,OI.

* Veau de la mère non adopté (Non adopted dam's calf).

** Une vache n'a pas senti son veau à la naissance; elle a par la suite refusé tout jeune ets'est tarie ( $A$ core did not smell her calf after birth, then she refused all young and dried up).

Témoin: présentation du second veau sans précaution lors d'une tétée du veau de la mère.

Masquage au vêlage : la vache est masquée pendant 8 jours à partir du vêlage,

- le veau de la mère et le deuxième veau sont enduits d'huile de pied de bouf, le veau de la mère de la naissance au huitième jour, le second veau du quatrième au huitième jour.

Masquage à 4 jours : la vache est masquée et les veaux sont enduits d'huile de pied de bœuf, du quatrième au huitième jour après le vêlage.

Untreated : foster calf presented without particular precaution when the dam suckled her owen calf.

Blindfolded at calving: the cow was blindfolded and her calf smeared with neats-foot oil for 8 days from calving, the foster calf teas smeared from the fth to the 8th day after calving.

Blindfolded the fourth day: the cow was blindfolded and the 2 calves smeared with neats-foot oil from the 4 th day to the 8 th day.

Les perturbations visuelles et olfactives pratiquées ont effectivement permis une augmentation importante de la proportion de veaux adoptés. Les faibles effectifs utilisés ne permettent cependant pas de préciser exactement l'importance de cette amélioration et demandent à être complétés.

Accepté pour publication en févier 1979.

\section{Summary}

Adoption of foster calves by Salers cor's several days after calving

In order to favour the adoption of calves by Salers cows 4 days after calving, we tried to disturb the cows by blinfolding them and smearing the calves with neatsfoot oil. Three groups of cows were compared:

I) The foster calf was presented without any treatment. 
2) The cow was blindfolded and her calf smeared with neatsfoot oil during eight days from calving; the foster calf was smeared from the 4 th to the 8 th day after calving.

3) The cow was blindfolded and the two calves smeared with neatsfoot oil from the $4^{\text {th }}$ to the 8th day.

The results of the third group are better than those of the two others. The methods used to counter-act the sense of sight and of smell favour the adoption of the foster calf.

\section{Références bibliographiques}

Crowley J. P., DARby T. F., I97I. A method of fostering calves for multiple suckling systems. Anim. Prod., 13, 382 (Abstr.).

Hudson S. J., I977. Multiple fostering of calves onto nurse cows at birth. App. Anim. Ethol., 3, $57-63$.

KILEY M., I976. Fostering and adoption in beef cattle. Brit. Cattle Breeders club Digest, $\mathrm{n}^{\circ} 3^{\mathrm{r}}$, 42-55.

LE NEINDRE P., GAREI J. P., I977. Eitude des relations nère-jeune chez les bovins domestiques: comparaison des liaisons existant entre la mère et des veaux légitimes ou adoptés. Biol. Behav., 2, 39-49.

LE NeINDre, P., PeTír M., Garei, J. P., i978. Allaitement de deux veaux par des vaches de race Salers. II. Adoption du deuxième veau. Ann. Zootech., 27, 553-569.

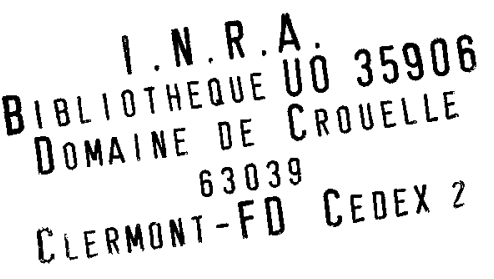

